\title{
Thymoquinone Induces Downregulation of BCR-ABL/JAK/ STAT Pathway and Apoptosis in K562 Leukemia Cells
}

\author{
Futoon Abedrabbu Al-Rawashde ${ }^{1}$, Wan Rohani Wan Taib ${ }^{1}$, Imilia Ismail ${ }^{1}$, \\ Muhammad Farid Johan², Abdullah Saleh Al-wajeeh ${ }^{3}$, Hamid Ali Nagi Al-Jamal ${ }^{1 *}$
}

\begin{abstract}
Objective: BCR ABL oncogene encodes the BCR-ABL chimeric protein, which is a constitutively activated non-receptor tyrosine kinase. The BCR-ABL oncoprotein is a key molecular basis for the pathogenesis of chronic myeloid leukemia (CML) via activation of several downstream signaling pathways including JAK/STAT pathway. Development of leukemia involves constitutive activation of signaling molecules including, JAK2, STAT3, STAT5A and STAT5B. Thymoquinone (TQ) is a bioactive constituent of Nigella sativa that has shown anticancer properties in various cancers. The present study aimed to evaluate the effect of TQ on the expression of BCR ABL, JAK2, STAT3, STAT5A and STAT5B genes and their consequences on the cell proliferation and apoptosis in K562 CML cells. Methods: BCR-ABL positive K562 CML cells were treated with TQ. Cytotoxicity was determined by Trypan blue exclusion assay. Apoptosis assay was performed by annexin V-FITC/PI staining assay and analyzed by flow cytometry. Transcription levels of BCR ABL, JAK2, STAT3, STAT5A and STAT5B genes were evaluated by reverse transcriptionquantitative polymerase chain reaction (RT-qPCR). Protein levels of JAK2 and STAT5 were determined by Jess Assay analysis. Results: TQ markedly decreased the cell proliferation and induced apoptosis in K562 cells $(\mathrm{P}<0.001)$ in a concentration dependent manner. TQ caused a significant decrease in the transcriptional levels of BCR ABL, JAK2, STAT3, STAT5A and STAT5B genes $(\mathrm{P}<0.001)$. TQ induced a significant decrease in JAK2 and STAT5 protein levels $(\mathrm{P}<0.001)$. Conclusion: our results indicated that TQ inhibited cell growth of K562 cells via downregulation of BCR ABL/ JAK2/STAT3 and STAT5 signaling and reducing JAK2 and STAT5 protein levels.
\end{abstract}

Keywords: Thymoquinone- CML- apoptosis- BCR ABL- STAT

Asian Pac J Cancer Prev, 22 (12), 3959- 3965

\section{Introduction}

Chronic myeloid leukemia (CML) is a hematopoietic stem cell malignancy characterized by the Philadelphia (Ph) chromosome (Rowley, 1973). The Ph chromosome is formed by a reciprocal translocation between Abelson murine leukemia (ABL) tyrosine kinase gene on chromosome 9 and breakpoint cluster region (BCR) tyrosine kinase gene on chromosome $22 \mathrm{t}(9 ; 22)$ (q34; q11), leading to the generation of the chimeric BCR ABL fusion gene (Rowley, 1973; Yu et al., 2020). The BCRABL oncogene encodes BCR-ABL chimeric protein, which is a key molecular basis for the pathogenesis of CML (Yu et al., 2020). The BCR-ABL oncoprotein has a constitutive kinase activity which induces myeloid cells proliferation through various downstream signaling pathways including the Janus activated kinase/signal transducer and activator of transcription (JAK/STAT) pathway (Braun et al., 2020).
STAT proteins are downstream targets of JAK that are essential transcription factors in the regulation of several cellular processes such as cell cycle, proliferation and apoptosis (Xin et al., 2020). JAK2 and STAT3 have been shown to act as an essential signaling molecules in the development of malignancy (Zhu et al., 2016). STAT5A and STAT5B have also been reported to play a role in the survival of cancer cells and pathogenesis of leukemia (Dalgıç et al., 2015). Constitutively activated STAT3 and STAT5 represent therapeutic targets for the treatment of BCR-ABL-driven leukemia (Mencalha et al., 2010; Berger et al., 2014; Al-Jamal et al., 2018a; Al-Jamal et al., 2018b).

The development of tyrosine kinase inhibitors (TKIs) against the BCR-ABL pathway has resulted in a significant advancements in CML treatment (Braun et al., 2020). However, resistance to TKI remains a significant challenge (Al-Jamal et al., 2018b; Maiti et al., 2020). Therefore, identifying additional significant therapies could have a substantial effect on CML treatment. 
Thymoquinone (TQ) is a phytochemical compound extracted from Nigella sativa (black seed) that was found to inhibit cancer cell growth and enhance apoptosis in several cancer cells, including myeloid leukemia cells (AlGhamdi et al., 2020; Almajali et al., 2021a). However, the effect mechanism of TQ on cancers still not fully clear.

However, the molecular mechanisms behind the inhibitory effect and the apoptotic activities of TQ in leukaemia are insufficiently explored. Therefore, this study aims to investigate the ability of TQ to downregulate BCR ABL, JAK2, STAT3, STAT5A and STAT5B and its effects on proliferation, and apoptosis of K562 CML cells. For that, RT-qPCR was conducted to evaluate the expression of BCR ABL, JAK2, STAT3, STAT5A and STAT5B, Jess Assay analysis was performed to evaluate the protein levels of JAK2 and STAT5, as well as cytotoxicity and apoptosis assays.

\section{Materials and Methods}

\section{Cell culture and reagents}

Human chronic myeloid leukemia cell line (K562) was purchased from the American Type Culture Collection (ATCC, Rockville, MD, USA). Roswell Park Memorial Institute medium (RPMI--1640), fetal bovine serum (FBS) and penicillin-streptomycin $(\mathrm{P} / \mathrm{S})$ were purchased from Elabscience Biotechnology Co., Ltd., (Wuhan, China). The cellswere cultured in complete RPMI medium with $10 \% \mathrm{v} / \mathrm{v}$ of FBS and $1 \% \mathrm{v} / \mathrm{v}$ penicillin-streptomycin. The cells were cultured in a humidified incubator (at $37^{\circ} \mathrm{C}, 5 \%$ $\mathrm{CO}_{2}$ ) and routinely subcultured when the cells reach $80 \%$ confluency at a density of $5 \times 10^{4}$ cells $/ \mathrm{ml}$.

\section{Thymoquinone treatment}

Thymoquinone (molecular formula: $\mathrm{C}_{10} \mathrm{H}_{12} \mathrm{O}_{2}$; molecular weight: 164.20 ) with purity $>98 \%$ was purchased from Sigma Aldrich (MO, USA). Dimethyl sulfoxide (DMSO) was purchased from Elabscience Biotechnology Co., Ltd., (Wuhan, China). TQ was dissolved with DMSO at a stock concentration of $20 \mu \mathrm{M}$, aliquoted and stored at $-80^{\circ} \mathrm{C}$. At the onset of treatment, TQ working concentrations were prepared with RPMI medium with a final DMSO concentration $<0.1 \%$.

Cell viability evaluation by Trypan blue exclusion assay

Trypan blue exclusion assay was used to measure the viability of K562 cells after TQ treatment. K562 cells were cultured into 12 -well plate at a density of $5 \times 10^{4}$ cells $/ \mathrm{ml}$. The cells were treated with $0 \mu \mathrm{M}, 3 \mu \mathrm{M}, 6 \mu \mathrm{M}, 9 \mu \mathrm{M}, 12$ $\mu \mathrm{M}, 15 \mu \mathrm{M}, 18 \mu \mathrm{M}, 21 \mu \mathrm{M}, 24 \mu \mathrm{M}, 27 \mu \mathrm{M}$ and $30 \mu \mathrm{M}$ of TQ and incubated at $37^{\circ} \mathrm{C}$ in a humidified incubator with $5 \% \mathrm{CO}_{2}$ for 24 hours. After the desired time of incubation, equal amounts of the cell suspension and Trypan blue solution $(0.2 \%$ in phosphate buffered saline) were mixed. The cells were counted using a hemocytometer (Reichert, USA) under an inverted microscope (Olympus, Tokyo, Japan). The experiment was performed in triplicate and the percentages of viability were presented relative to the controls (untreated cells, 100\%).
Apoptosis analysis by annexin V-FITC/propidium iodide (PI) staining

Annexin V-FITC/ PI apoptosis detection kit (Nacalai Tesque, INC., Kyoto, JAPAN) was used to measure the K562 cells apoptosis rate. The cells were treated with $12 \mu \mathrm{M}, 18 \mu \mathrm{M}, 24 \mu \mathrm{M}, 27 \mu \mathrm{M}$ and $30 \mu \mathrm{M}$ of TQ for 24 hours. The cells were washed twice with PBS then resuspended into Annexin $\mathrm{V}$ binding buffer to a final concentration of $1 \times 10^{6}$ cells $/ \mathrm{ml}$. Then, $5 \mu$ of Annexin V-FITC conjugate and $5 \mu \mathrm{l}$ of PI solution were added to $100 \mu \mathrm{l}$ of the cell suspension and incubated in the dark for 15 minutes at room temperature. The cells solution was then diluted with $400 \mu \mathrm{l}$ of Annexin V binding buffer. The apoptosis measurements were performed using CytoFLEX flow cytometer (Beckman Coulter, USA) and 10,000 events were acquired and analyzed using CytExpert for CytoFLEX Acquisition and Analysis Software (Beckman Coulter, USA).

\section{RNA Extraction}

K562 cells at a density of $5 \times 10^{4}$ cells $/ \mathrm{ml}$ were treated with $15 \mu \mathrm{M}$ of TQ for 48 hours. Total RNA was extracted from treated and untreated cells using ReliaPrep ${ }^{\mathrm{TM}} \mathrm{RNA}$ Cell Miniprep System RNA extraction kit (Promega, USA) according to the manufacturer's protocol. The Nanodrop Photometer (Implen, Weslake Village, USA) was used to evaluate the concentration and purity of the extracted RNA.

\section{Gene expression evaluation by RT-qPCR}

Total RNA (100 ng) was reverse transcribed into cDNA using GoTaq ${ }^{\circledR}$ 2-Step RT-qPCR System kit (Promega, USA) according to the manufacturer's protocol. Then gene expression of BCR-ABL, JAK2, STAT3, STAT5A and STAT5B was investigated using SYBR Green-based GoTaq 2-Step RT-qPCR System kit (Promega, USA) following the manufacturer's protocol. The PCR reaction was performed in triplicates with the following cycling parameters: activation step, 2 minutes at $95^{\circ} \mathrm{C}$; denaturation step, 40 cycles for 15 seconds at $95^{\circ} \mathrm{C}$; annealing and extension, 40 cycles at for 1 minute at $60^{\circ} \mathrm{C}$. Reverse transcription-quantitative polymerase chain reaction (RT-qPCR) was performed using Applied Biosystem StepOnePlus ${ }^{\mathrm{TM}}$ thermocycler (Applied Biosystems, Foster City, CA, USA). The primers sequences used for the PCR reactions are presented in Table 1. StepOne Software v2.3 (ABI step one plus, USA) was used to analyse the data. The expression fold changes $\left(2^{-\Delta \Delta \mathrm{Ct}}\right)$ were calculated after normalization with a reference gene ( $\beta$-actin).

\section{Preparation of total cell lysates}

K562 leukemia cells were treated with $15 \mu \mathrm{M}$ of TQ for 48 hours. Total cell lysates were obtained from treated and untreated cells using RIPA buffer (containing $50 \mathrm{mmol} / \mathrm{l}$ Tris-HCl buffer (PH 7.6), $150 \mathrm{mmol} / \mathrm{l} \mathrm{NaCl}$, $1 \%$ Nonidet ${ }^{\mathbb{R}} \mathrm{P}-40$ Substitute, $0.5 \%$ sodium deoxycholate, $0.1 \%$ SDS, and $1 \times$ protease inhibitor cocktail) (Nacalai Tesque, Kyoto, JAPAN) following the manufacturer's protocol. Bradford protein assay (Kielkopf et al., 2020) was used to measure protein concentrations using Coomassie brilliant blue (CBB) and fetal bovine serum 
(FBS) from Nacalai tesque, Inc. (Kyoto, Japan). The absorbance was measured at $495 \mathrm{~nm}$ by microplate reader (Infinite M200, Tecan, Männedorf, Switzerland).

Evaluation of protein expression using Jess simple western analysis

Protein expression was evaluated by capillary-based western analysis using Protein Simple Jess system (Jess; Protein Simple, CA, USA) following the manufacturer's protocol. Briefly, sample buffer was used to dilute the cell lysates ( $1 \mathrm{mg} / \mathrm{ml}$ concentration). Master mix, (40 $\mathrm{mM}$ dithiothreitol, $1 \times$ sample buffer and $1 \times$ fluorescent standard) was added to each sample and heated $\left(95^{\circ} \mathrm{C}\right.$, 5 minutes) to denature the samples. Subsequently, $3 \mu 1$ of the denatured proteins, $10 \mu \mathrm{l}$ of each of the primary antibodies (at 1:20 dilution), HRP-conjugated anti-mouse secondary antibodies, protein normalization solution and chemiluminescent substrate were loaded into the desired wells of the assay plate. For each assay, a biotinylated ladder cartridge provided molecular weight standard $(12-230 \mathrm{kDa})$ was incorporated. The plate and capillaries were placed into the Jess machine for automated protein electrophoresis, blocking, antibody incubation and signal detection. To account for any variations in protein loading, total protein was normalised within capillaries. Compass software (ProteinSimple) was used to analyse chemiluminescent reactions. The following primary antibodies were used: Anti-JAK2 (NBP2-59451; Novus Biologicals, LLC, Littleton, CO, USA), anti-STAT5 (AF2168; R\&D System, Minneapolis, USA).

Statistical Analysis

Data analyses were conducted using the Statistical Package for Social Sciences (SPSS) version 25 (SPSS Inc., Chicago, USA). The data between TQ-treated and untreated K562 cells were analysed using Wilcoxon signed-rank test and repeated measures ANOVA with Bonferroni correction with the significant level set at $\mathrm{p}<$ $0.05, \mathrm{p}<0.01$, and $\mathrm{p}<0.001$.

\section{Results}

$T Q$ inhibits $K 562$ cells proliferation

Trypan blue exclusion assay was used to investigate
Thymoquinone Downregulates BCR-ABL/JAK-STAT Signaling the cells viability . K562 cells were incubated with serial concentrations of TQ $(0 \mu \mathrm{M}, 3 \mu \mathrm{M}, 6 \mu \mathrm{M}, 9 \mu \mathrm{M}, 12 \mu \mathrm{M}$, $15 \mu \mathrm{M}, 18 \mu \mathrm{M}, 21 \mu \mathrm{M}, 24 \mu \mathrm{M}, 27 \mu \mathrm{M}$ and $30 \mu \mathrm{M})$. The results indicated a dose dependent effect of TQ on cell viability, with IC50 of $23 \pm 5.2 \mu \mathrm{M}$ after 24 hours of treatment (Figure 1).

\section{$T Q$ induces apoptosis in $\mathrm{K} 562$ cells}

After exposing K562 cells to several concentrations of TQ $(12 \mu \mathrm{M}, 18 \mu \mathrm{M}, 24 \mu \mathrm{M}, 27 \mu \mathrm{M}$ and $30 \mu \mathrm{M})$ for 24 hours, Annexin-V/FITC/PI apoptosis detection assay was conducted to assess the apoptosis rates. As shown in Figure 2A, the viable cells in the lower left quadrant were stained negative with both annexin V -FITC and PI. Early apoptotic cells can be detected in the lower right quadrant and were stained positive with annexin V-FITC and negative with PI, so they can be distinguished from late apoptotic cells in the upper right quadrant which were

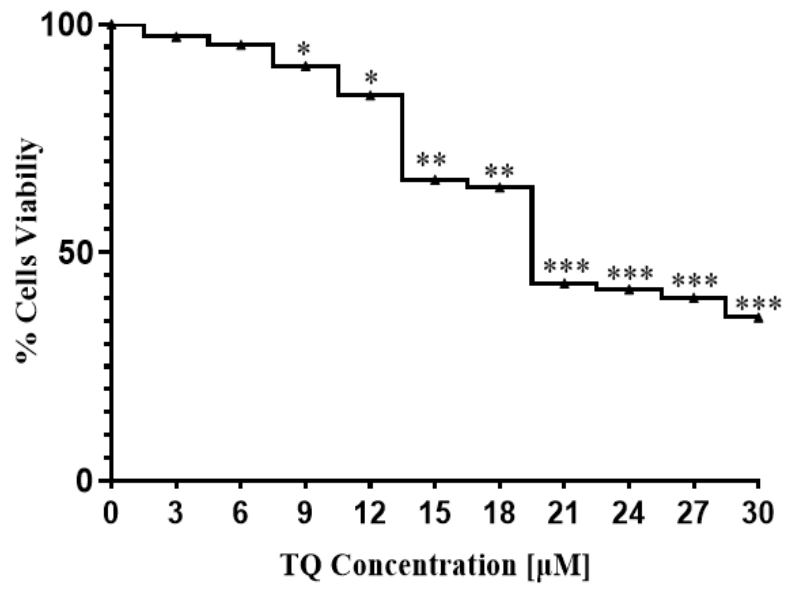

Figure 1. Dose-Dependent Inhibitory Effects of TQ on the Proliferation of K562 Cells. Trypan blue exclusion assay was used to assess the growth inhibitory effects of different concentrations of TQ $(0 \mu \mathrm{M}, 3 \mu \mathrm{M}, 6 \mu \mathrm{M}$, $9 \mu \mathrm{M}, 12 \mu \mathrm{M}, 15 \mu \mathrm{M}, 18 \mu \mathrm{M}, 21 \mu \mathrm{M}, 24 \mu \mathrm{M}, 27 \mu \mathrm{M}$ and $30 \mu \mathrm{M})$ on K562 cells after 24 hours of treatment, the IC50 value was $23 \pm 4.3 \mu \mathrm{M}$. Data were stated as percentages of viable cells relative to the untreated cells. The values were presented as mean $\pm \mathrm{SD}$ of three independent experiments. ${ }^{*} \mathrm{p}<0.05, * * \mathrm{p}<0.01, * * * \mathrm{p}<0.00$

Table 1. Primer Sequences for RT-qPCR Assay

\begin{tabular}{|c|c|c|}
\hline Genes & Primer sequence $\left(5^{\prime}-3^{\prime}\right)$ & Reference \\
\hline \multirow[t]{2}{*}{$B C R-A B L$} & Forward: TCCGCTGACCATCAATAAGGA & (Marega et al., 2010) \\
\hline & Reverse: CACTCAGACCCTGAGGCTCAA & \\
\hline \multirow[t]{2}{*}{$J A K 2$} & Forward: TGTCTTACCTCTTTGCTCAGTGGCG & (Gupta et al., 2012) \\
\hline & Reverse: CAATGACATTTTCTCGCTCGACAGC & \\
\hline \multirow[t]{2}{*}{ STAT3 } & Forward GATTGACCAGCAGTATAGCCGCTTC & (Bellon et al., 2018) \\
\hline & Reverse: CTGCAGTCTGTAGAAGGCGTG & \\
\hline \multirow[t]{2}{*}{ STAT5A } & Forward: GAAGCTGAACGTGCACATGAATC & (Dalgıç et al., 2015) \\
\hline & Reverse: GTAGGGACAGAGTCTTCACCTGG & \\
\hline \multirow[t]{2}{*}{ STAT5B } & Forward: AGTTTGATTCTCAGGAAAGAATGT & (Dalgıç et al., 2015) \\
\hline & Reverse: TCCATCAACAGCTTTAGCAGT & \\
\hline \multirow[t]{2}{*}{$\beta$-actin } & Forward: CTGGCACCCAGGACAATG & (Relles et al., 2016) \\
\hline & Reverse: GCCGATCCACACGGAGTA & \\
\hline
\end{tabular}



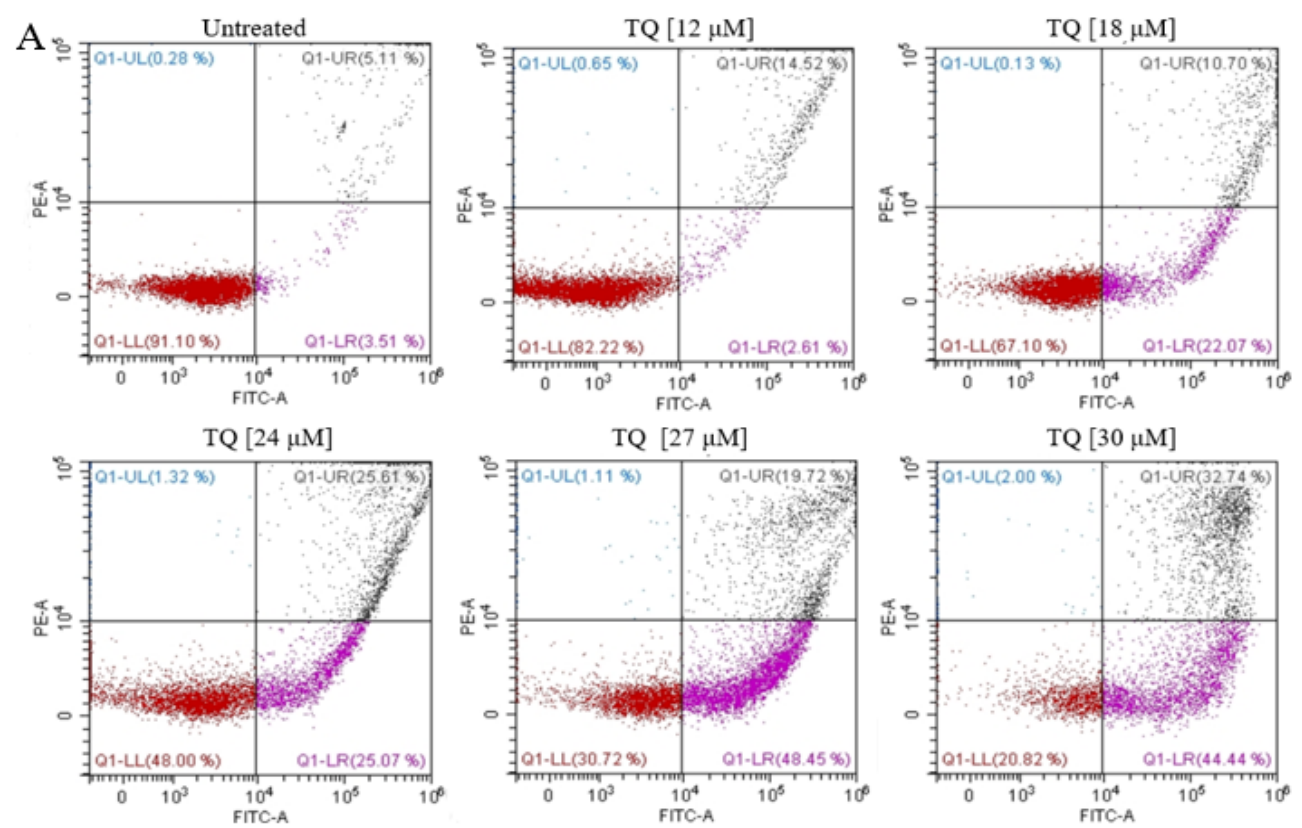

B

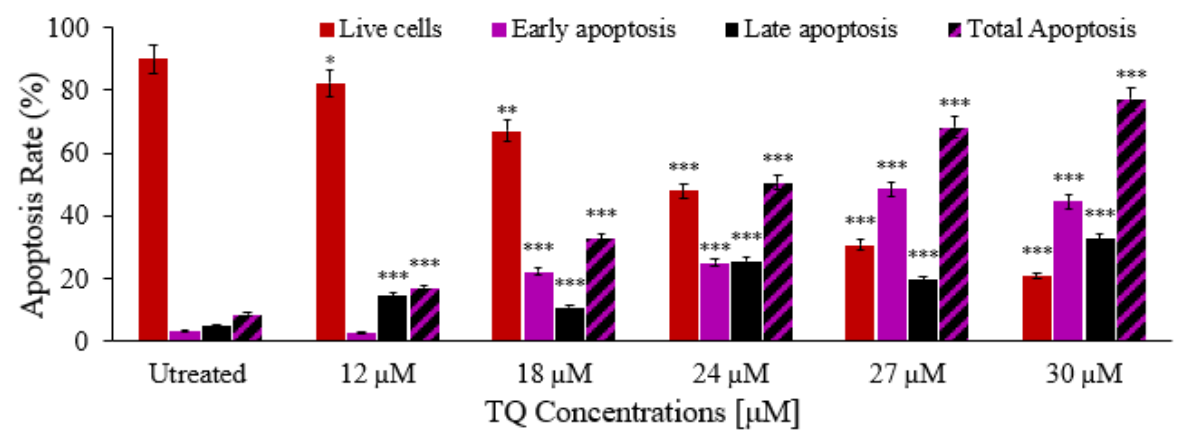

Figure 2. Dose-Course Flow Cytometry Analysis of K562 Cells after TQ Treatment. (A) Cells were treated with 12 $\mu \mathrm{M}, 18 \mu \mathrm{M}, 24 \mu \mathrm{M}, 27 \mu \mathrm{M}$ and $30 \mu \mathrm{M}$ of TQ for 24 hours. The apoptosis events in K562 cells were assessed using Annexin V-FITC and PI- double stain. (B) The bar graph is the percentages of cells relative to the untreated cells after 24 hours of treatment. Values were presented as means $\pm \mathrm{SD}$ of three independent experiments. ${ }^{*} \mathrm{p}<0.05,{ }^{*} \mathrm{p}<0.01$, $* * * \mathrm{p}<0.001$

stained positive with both annexin V -FITC and PI. The apoptotic K562 cells were increased by increasing TQ concentration (Figure 2A and 2B). The total apoptotic cells accounted for $17.13 \%, 32.77 \%, 50.68 \%, 68.17 \%$ and $77.18 \%$ after treatment with $12 \mu \mathrm{M}, 18 \mu \mathrm{M}, 24 \mu \mathrm{M}$,
$27 \mu \mathrm{M}$ and $30 \mu \mathrm{M} \mathrm{TQ}$, respectively for 24 hours.

TQ downregulates BCR-ABL, JAK2 and STATs in K562 cells

The expression of BCR-ABL, JAK2, STAT3, STAT5A

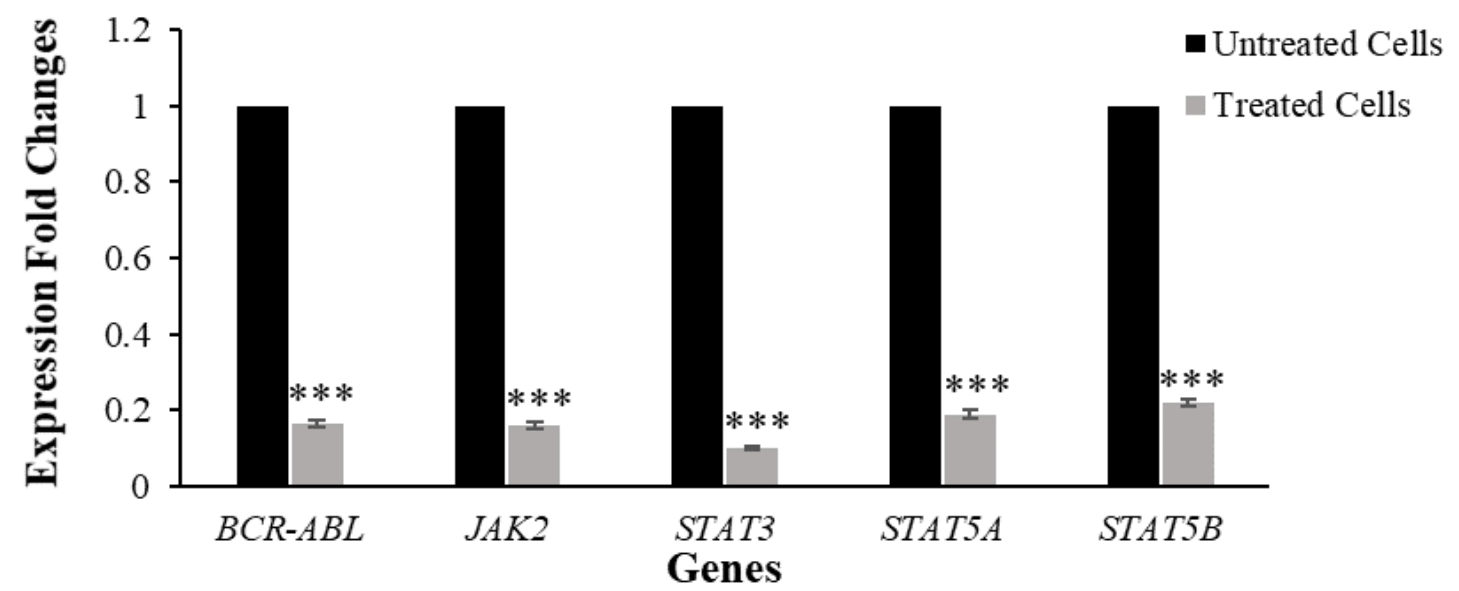

Figure 3. Effect of TQ on the mRNA expression of BCR-ABL, JAK2, STAT3, STAT5A and STAT5B in K562 cells. RT-qPCR analysis were performed in triplicate before and after treatment with $15 \mu \mathrm{M}$ of TQ for 48 hours. The graph showed the down-regulation of BCR-ABL, JAK2, STAT3, STAT5A and STAT5B. Wilcoxon signed rank test was conducted and values were stated as median (interquartile range); $* * * p<0.001$. 

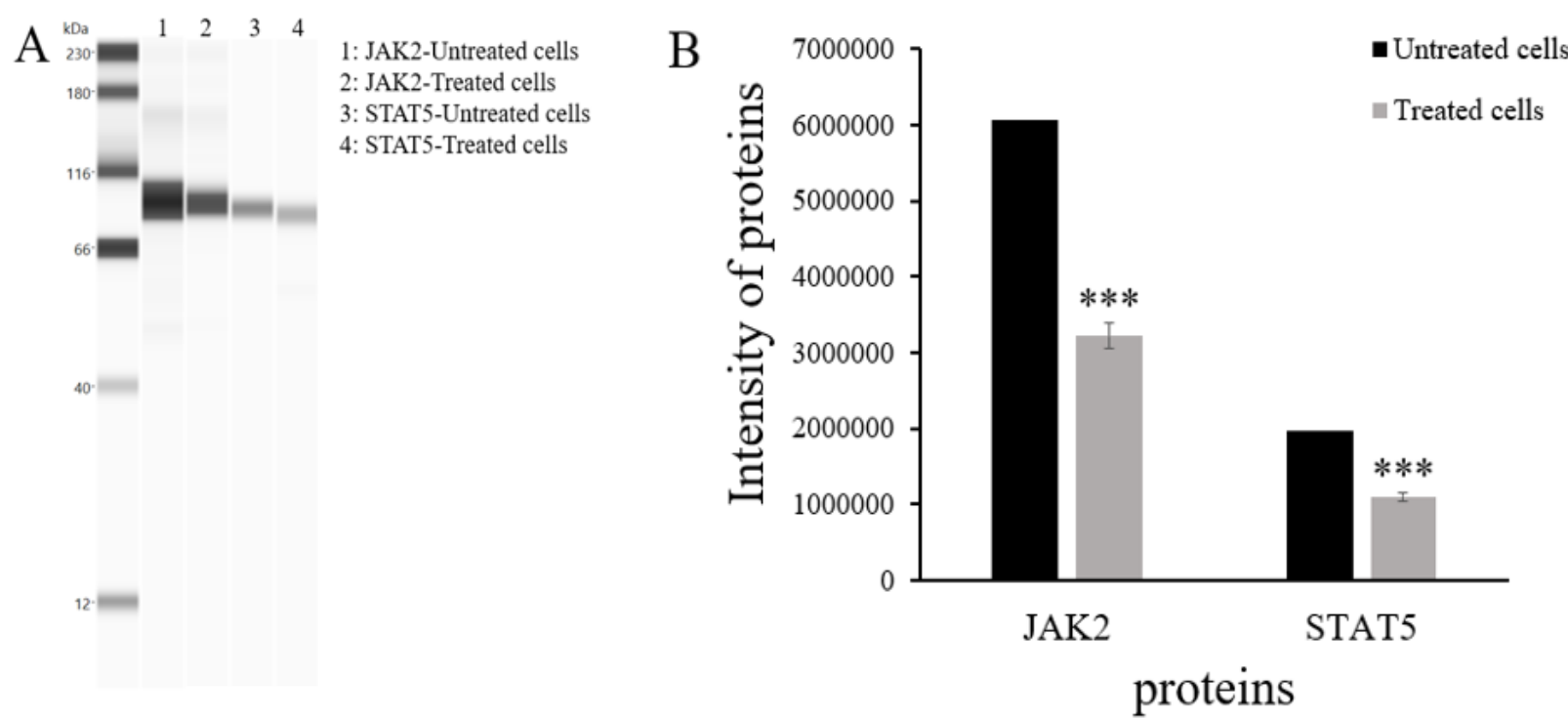

Figure 4. Effect of TQ on JAK2 and STAT5 Protein Levels in K562 Cells. Jess simple western analysis was performed in triplicate. The cells were treated with $15 \mu \mathrm{M}$ of TQ for 48 hours. (A) Representative image of JAK2 and STAT5 protein levels from Jess simple western blotting. (B) Bar graph shows the reduction of JAK2 and STAT5 protein levels in TQ-treated K562 cells. Wilcoxon signed rank test was conducted and values were stated as median (interquartile range); $* * * \mathrm{p}<0.001$.

and STAT5B genes was investigated in K562 cells using RT-qPCR The cells were treated with $15 \mu \mathrm{M}$ of TQ for 48 hours. The results of RT-qPCR showed a significant decrease in mRNA expression levels of BCR-ABL, JAK2, STAT3, STAT5A and STAT5B (Figure 3). The expression of BCR-ABL and JAK2 was dramatically decreased by 6 -fold $(\mathrm{P}<0.001)$ and 6.4-fold $(\mathrm{P}<0.001)$, respectively after TQ treatment compared to untreated cells. The expression STAT3, STAT5A and STAT5B was also significantly decreased by 4.7 -fold $(\mathrm{P}<0.001), 5.4-$ fold $(\mathrm{P}<0.001)$ and 4.5 -fold $(\mathrm{P}<0.001)$, respectively compared to untreated cells.

$T Q$ reduces JAK2 and STAT5 protein levels in K562 cells The protein levels of JAK2 and STAT5 were evaluated by Jess simple western analysis in K562 cells. The cells were exposed to $15 \mu \mathrm{M}$ of TQ and incubated for 48 hours. TQ treatment significantly reduced JAK2 and STAT5 protein levels as compared to untreated cells $(\mathrm{P}<0.001)$, Figure 4.

\section{Discussion}

CML is a myeloproliferative neoplasm caused by a constitutively activated BCR-ABL tyrosine kinase (Yu et al., 2020). Dysregulation of JAK/STAT signalling plays an essential role in the pathogenesis of CML (Braun et al., 2020). BCR-ABL activates many pro-oncogenic signaling molecules including JAK2, STAT3 and STAT5, which induce cell proliferation with inhibition of cell differentiation and apoptosis (Ilaria and Van Etten, 1996; Braun et al., 2020). TKIs are currently being used to treat CML and have been shown to be clinically efficient (Braun et al., 2020). However, TKIs effectiveness was temporarily and the emergence of TKI resistance represents a substantial challenge (Al-Jamal et al., 2018b;
Maiti et al., 2020).

TQ has been reported to have anti-cancer activities in variety of cancer cells (Houssein et al., 2020; Almajali et al., 2021a). However, the molecular mechanisms underlying TQ anticancer potential effectiveness are not fully investigated. Therefore, the purpose of this study was to elucidate the effects of TQ on the expression of BCR-ABL and genes involved in JAK/STAT signaling with their consequences on the protein levels of JAK2 and STAT5 in K562 leukemia cells.

The findings of this study showed that TQ potentially induced anti-proliferative effect on BCR-ABL positive K562 CML cells in a dose dependent manner. As shown in Figure 1, the viability of the cells was decreased gradually from $100 \%$ to be only $35 \%$ viable cells by increasing the concentration of TQ to $30 \mu \mathrm{M}$ and incubation for 24 hours. These findings were consisted with that previously reported in which TQ inhibited the proliferation of K562, Jurkat and HL60 leukemia cells (AlGhamdi et al., 2020; Almajali et al., 2021b).

In the present study, BCR-ABL along with key downstream molecules of BCR-ABL pathway were investigated in K562 leukemia cells. The findings showed that TQ significantly reduced the expression of BCR-ABL (6 times lower) in K562 treated cells compared to untreated cells $(\mathrm{P}<0.001)$. This finding was similar to what has been reported previously in which BCR-ABL mRNA level was decreased in K562 cells after OGP46 treatment (Wei et al., 2020). Additionally, the results of the present study revealed that TQ induced a significant downregulation in the expression of JAK2 by 6.4-fold $(\mathrm{P}<0.001)$, STAT3 by 4.7 -fold $(\mathrm{P}<0.001)$, STAT5A by 5.3-fold $(\mathrm{P}<0.001)$ and STAT5B by 4.5 -fold $(\mathrm{P}<0.001)$ compared to untreated cells. These findings were similar to others' findings in which JAK2 was downregulated in HepG2 and MHCC97-H hepatocellular carcinoma cells Asian Pacific Journal of Cancer Prevention, Vol 223963 
after scutellarin treatment (Liu et al., 2019), STAT3 was downregulated in HGC27, BGC823, and SGC7901 gastric cancer cell lines after TQ treatment (Zhu et al., 2016), STAT5A and STAT5B were downregulated in K562 cells after dasatinib treatment (Dalgıç et al., 2015).

Furthermore, JAK2 protein intensity was reduced in HepG2 and MHCC97-H hepatocellular carcinoma cells after scutellarin treatment (Liu et al., 2019). STAT5 protein intensity was also lowered in K562 cells after treatment with Trifolium Repens extract compared to the untreated control (Sarno et al., 2020). In similarity, the results of the present study revealed a significant reduction in the intensity of JAK2 and STAT5 proteins $(p<0.001)$ in TQ treated K562 cells compared to untreated cells. On the other hand, the results were inconsistent with previous study that shown STAT5 protein intensity was not affected by TQ treatment (Zhu et al., 2016).

Furthermore, apoptosis is the main mechanism of the programmed cell death and inhibited apoptosis is associated with the development of cancer (Jiang et al., 2021). BCR-ABL and activated JAK/STAT signaling have been found to exert anti apoptotic effects in CML cells (Chen et al., 2016; Jiang et al., 2021). Additionally, TQ had induced apoptosis in U87MG human Glioblastoma multiforme cells (Khazaei and Pazhouhi, 2017) and CEM and Jurkat human acute lymphoblastic leukemia cells (Soltani et al., 2017; Houssein et al., 2020). Furthermore, the findings of the current study showed that TQ significantly induced apoptosis in K562 cells in a dose dependent manner. As shown in Figure 2A and 2B, the percentage of apoptosis was increased significantly from $17.13 \%$ to $77.18 \%$ apoptotic cells by increasing TQ concentration from $12 \mu \mathrm{M}$ to $30 \mu \mathrm{M}$, respectively after 24 hours of treatment.

Taken together, our findings suggest that TQ effectively inhibited the growth of BCR-ABL positive K562 CML cells with apoptosis induction through downregulation of BCR-ABL, JAK2, STAT3, STAT5A and STAT5B and reducing JAK2 and STAT5 protein levels.

In conclusion, the findings indicated that TQ potentially inhibited K562 cells proliferation and enhanced apoptosis through suppression of BCR-ABL, JAK2, STAT5A and STATB genes suggesting that TQ might be a promising candidate for the treatment of CML patients. However, further investigations are needed to clarify the effect of TQ on signaling pathways.

\section{Author Contribution Statement}

Main manuscript writing and investigations H.A. and F.A.; data collection, analysis and curation H.A. and F.A.; interpretation of the results and statistical analysis H.A and F.A.; software and manuscript revision, F.A., H.A., W.I, I.I., M.J.,A.S.W; supervision and funding acquisition H.A.

\section{Acknowledgements}

The authors would like to thank everyone at UniSZA's Centralized Laboratory Management Center and Medical School's Cell Culture and Molecular Laboratories for their assistance.
Funding Statement

This study was funded by the Fundamental Research Grant Scheme of the Ministry of Education, Malaysia [FRGS/1/2019/SKK08/UNISZA/02/3 (RR330)].

Statement conflict of Interest

The authors declare no competing interests.

\section{References}

Al-Jamal HAN, Johan MF, Jusoh SAM, et al (2018a). Re-expression of bone marrow proteoglycan-2 by 5-azacytidine is associated with STAT3 inactivation and sensitivity response to imatinib in resistant CML cells. Asian Pac J Cancer Prev, 19, 1585.

Al-Jamal HAN, Taib WRW, Jusoh SAM, et al (2018b). Azacytidine Enhances Sensitivity Response to Imatinib in BCR/ABL positive CML Cell Line. JBCS, 2, 20-2.

AlGhamdi AA, Mohammed MRS, Zamzami MA, et al (2020). Untargeted metabolomics identifies key metabolic pathways altered by thymoquinone in leukemic cancer cells. Nutrients, 12, 1792.

Almajali B, Al-Jamal HAN, Taib WRW, et al (2021a). Thymoquinone, as a novel therapeutic candidate of cancers. Pharmaceuticals, 14, 369.

Almajali B, Al-Jamal HAN, Taib WRW, et al (2021b). Thymoquinone Suppresses Cell Proliferation and Enhances Apoptosis of HL60 Leukemia Cells through Re-Expression of JAK/STAT Negative Regulators. Asian Pac J Cancer Prev, 22, 879 .

Bellon M, Moles R, Chaib-Mezrag H, et al (2018). JAG1 overexpression contributes to Notch 1 signaling and the migration of HTLV-1-transformed ATL cells. J Hematol Oncol, 11, 1-12.

Berger A, Sexl V, Valent P, et al (2014). Inhibition of STAT5: a therapeutic option in BCR-ABL1-driven leukemia. Oncotarget, 5, 9564.

Braun TP, Eide CA, Druker BJ (2020). Response and resistance to BCR-ABL1-targeted therapies. Cancer Cell, 37, 530-42.

Chen C-W, Lee Y-L, Liou J-P, et al (2016). A novel tubulin polymerization inhibitor, MPT0B206, downregulates $\mathrm{Bcr}-\mathrm{Abl}$ expression and induces apoptosis in imatinibsensitive and imatinib-resistant CML cells. Apoptosis, 21, 1008-18.

Dalgıç CT, Kaymaz BT, Özkan MC, et al (2015). Investigating the role of JAK/STAT pathway on dasatinib-induced apoptosis for CML cell model K562. Clin Lymphoma Myeloma Leuk, 15, 161-6.

Gupta M, Han JJ, Stenson M, et al (2012). Elevated serum IL-10 levels in diffuse large B-cell lymphoma: a mechanism of aberrant JAK2 activation. Blood, 119, 2844-53.

Houssein M, Fatfat M, Habli Z, et al (2020). Thymoquinone synergizes with arsenic and interferon alpha to target human T-cell leukemia/lymphoma. Life Sci, 251, 117639.

Ilaria RL, Van Etten RA(1996). P210 and P190BCR/ABL induce the tyrosine phosphorylation and DNA binding activity of multiple specific STAT family members. J Biol Chem, 271, 31704-10.

Jiang S, Wang X, He Y, et al (2021). Suppression of USP7 induces BCR-ABL degradation and chronic myelogenous leukemia cell apoptosis. Cell Death Dis, 12, 1-12.

Khazaei M, Pazhouhi M (2017). Temozolomide-mediated apoptotic death is improved by thymoquinone in U87MG cell line. Cancer Investig, 35, 225-36.

Kielkopf CL, Bauer W, Urbatsch IL (2020). Bradford assay for determining protein concentration. Cold Spring Harb. 
Protoc, 2020, pdb. prot102269.

Liu K, Tian T, Zheng Y, et al (2019). Scutellarin inhibits proliferation and invasion of hepatocellular carcinoma cells via down-regulation of JAK2/STAT3 pathway. $J$ Cell Mol Med, 23, 3040-4.

Maiti A, Franquiz MJ, Ravandi F, et al (2020). Venetoclax and BCR-ABL tyrosine kinase inhibitor combinations: outcome in patients with philadelphia chromosome-positive advanced myeloid leukemias. Acta Haematol, 143, 513-9.

Marega M, Piazza R, Pirola A, et al (2010). BCR and BCR-ABL regulation during myeloid differentiation in healthy donors and in chronic phase/blast crisis CML patients. Leukemia, 24, 1445-9.

Mencalha AL, Du Rocher B, Salles D, et al (2010). LLL-3, a STAT3 inhibitor, represses BCR-ABL-positive cell proliferation, activates apoptosis and improves the effects of Imatinib mesylate. Cancer Chemother Pharmacol, 65, 1039-46.

Relles D, Chipitsyna GI, Gong Q, et al (2016). Thymoquinone promotes pancreatic cancer cell death and reduction of tumor size through combined inhibition of histone deacetylation and induction of histone acetylation. Adv Prev Med, 2016.

Rowley JD (1973). A new consistent chromosomal abnormality in chronic myelogenous leukaemia identified by quinacrine fluorescence and Giemsa staining. Nature, 243, 290-3.

Salim LZA, Mohan S, Othman R, et al (2013). Thymoquinone induces mitochondria-mediated apoptosis in acute lymphoblastic leukaemia in vitro. Molecules, 18, 11219-40.

Sarno F, Pepe G, Termolino P, et al (2020). Trifolium Repens blocks proliferation in chronic myelogenous leukemia via the BCR-ABL/STAT5 pathway. Cells, 9, 379.

Soltani A, Pourgheysari B, Shirzad H, et al (2017). Antiproliferative and apoptosis-inducing activities of thymoquinone in lymphoblastic leukemia cell line. Indian J Hematol Blood Transfus, 33, 516-24.

Wei L, Yang Y, Gupta P, et al (2020). A small molecule inhibitor, OGP46, is effective against imatinib-resistant BCR-ABL mutations via the BCR-ABL/JAK-STAT pathway. Mol Ther Oncolytics, 18, 137-48.

Xin P, Xu X, Deng C, et al (2020). The role of JAK/STAT signaling pathway and its inhibitors in diseases. Int Immunopharmacol, 80, 106210.

Yu C, Gorantla SP, Müller-RudorfA, et al (2020). Phosphorylation of BECLIN-1 by BCR-ABL suppresses autophagy in chronic myeloid leukemia. Haematologica, 105, 1285.

Zhu W-Q, Wang J, Guo X-F, et al (2016). Thymoquinone inhibits proliferation in gastric cancer via the STAT3 pathway in vivo and in vitro. World J Gastroenterol, 22, 4149.

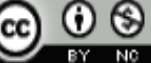

This work is licensed under a Creative Commons AttributionNon Commercial 4.0 International License. 